\title{
Simple and Safe Foley Catheter-Guided Tracheostomy Tube Changes
}

\author{
Chan Yong Park, Wu Seong Kang \\ Department of Trauma Surgery, Wonkwang University, Jeonbuk, Korea
}

The operator burden and the possible risks are unknown when performing first tracheostomy tube changes in patients with tracheostomy. Therefore, the authors would like to introduce a very simple and easy method to reduce the operator burden performing first tracheostomy tube changes to ensure patient safety.

(Trauma Image Proced 2018(2):72-75)

Key Words: First tracheostomy tube change; Simple; Safety

\section{CASE}

A 66-year-old male was admitted to our hospital with severe trauma who underwent tracheostomy using a 7.5-mm Cuffed Tracheostomy Tube. He underwent first tracheostomy tube change 14 days later. The authors named this method "Foley Catheter-Guided Tracheostomy Tube Change." The details are as follows:

\section{The "Foley Catheter-Guided Tracheostomy Tube Change" Method}

\section{Preparation (Fig. 1)}

1) Prepare a new tracheostomy tube, 10-cc syringe, scissors, gauze, and lubricant.

2) Prepare a Foley catheter of appropriate thickness to insert in the tracheostomy tube.

For example:

7.5-mm tracheostomy tube using $\geq 20$-Fr Foley catheter 7.0-mm tracheostomy tube $\geq 18$-Fr Foley catheter

3) To use the Foley catheter as a guide, cut off the thick part at the backside with scissors.

4) Pre-lubricate the end of the tracheostomy tube and the Foley catheter.

2. Foley catheter-guided tracheostomy tube removal (Fig. 2)

1) Perform deballooning of the patient's tracheostomy tube and release the fixation.

2) Separate the ventilator circuit from the tracheostomy tube.

3) Suction the tracheal and oral secretions.

4) Remove the tracheostomy tube while keeping the scissored Foley catheter in the tracheostomy tube. (In most cases, a large amount of secretion is released.)

Received: October 23, 2018 Revised: November 10, 2018 Accepted: November 12, 2018

Correspondence to: Chan Yong Park, Department of Trauma Surgery, Wonkwang University Hospital, Jeonbuk, Korea

Tel: 82-63-859-2602, Fax: 82-63-859-2029, E-mail: wkafyddı@hanmail.net

Copyright (c) 2018 Korean Association for Research, Procedures and Education on Trauma. All rights reserved.

@This is an open-access article distributed under the terms of the Creative Commons Attribution Non-Commercial License (http://creativecommons.org/ licenses/by-nc/4.0) which permits unrestricted noncommercial use, distribution, and reproduction in any medium, provided the original work is properly cited 


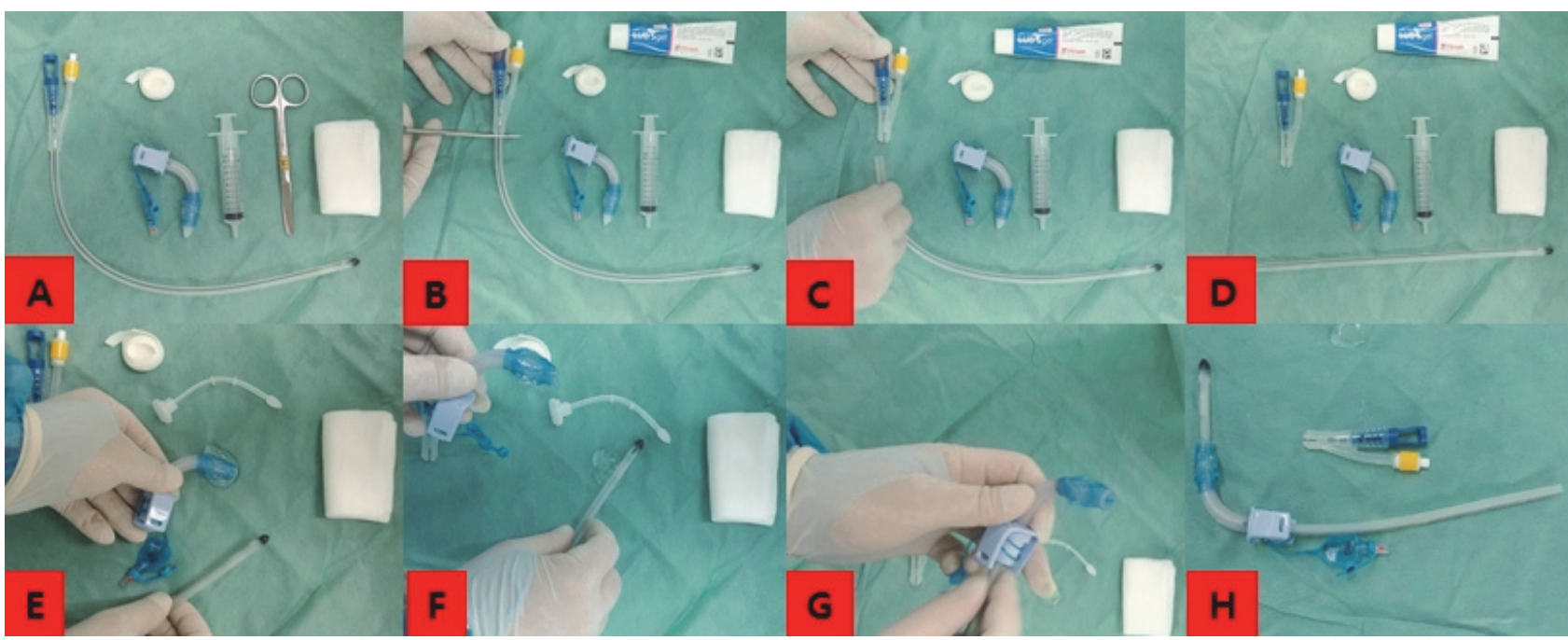

Fig. 1. Preparation

Prepare a new tracheostomy tube, 10-cc syringe, scissors, gauze, and lubricant. Prepare a Foley catheter of appropriate thickness to insert the tracheostomy tube. To use the Foley catheter as a guide, cut off the thick part of the backside with scissors. Pre-lubricate the end of the tracheostomy tube and the Foley catheter.

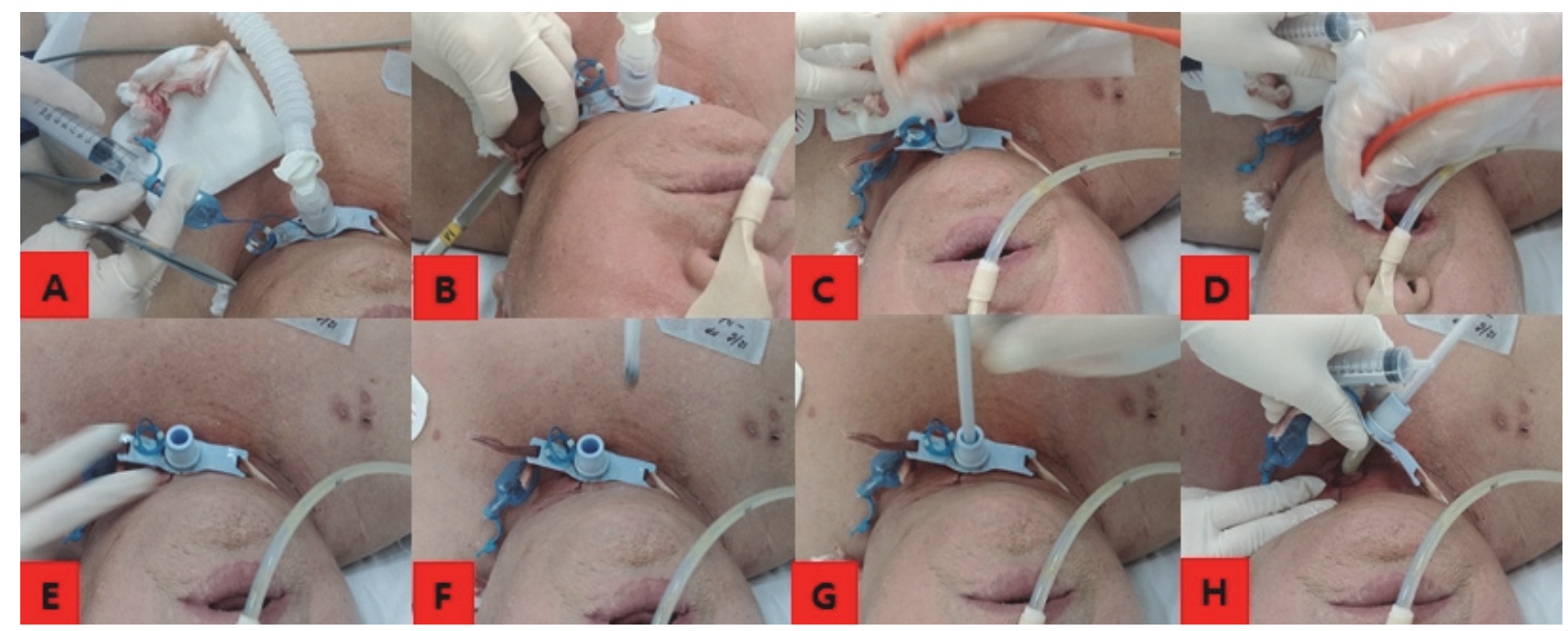

Fig. 2. Foley catheter-guided tracheostomy tube removal

Perform deballoning of the patient' $s$ tracheostomy tube and release the fixation. Separate the ventilator circuit from the tracheostomy tube. Suction the tracheal and oral secretions. Remove the tracheostomy tube while keeping the scissored Foley catheter in the tracheostomy tube.

\section{Foley catheter-guided tracheostomy tube insertion} (Fig. 3)

1) Be extra careful not to let the Foley catheter come out.

2) Hold the back part with gauze so that the whole of Foley catheter does not enter into the tract.

3) Use the Foley catheter as a guide, carefully insert the tracheostomy tube to be replaced. (Because this is the first tracheostomy tube change, some resistance may be felt in the tract.)

4) Balloon and fix the tracheostomy tube.

5) Connect the ventilator and check the breath sounds with the stethoscope. (Make sure no other problems occur.) 


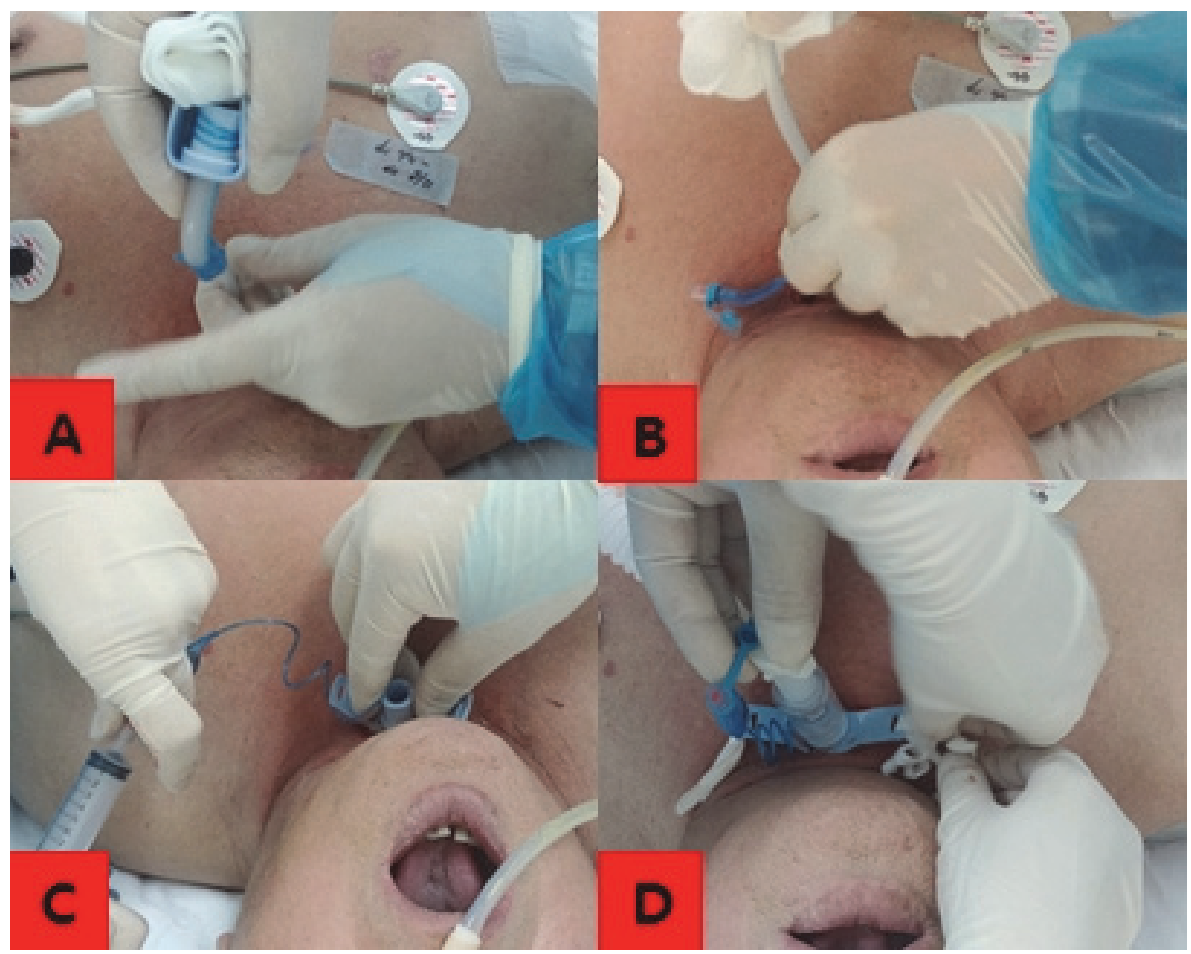

Fig. 3. Foley catheter-guided tracheostomy tube insertion Use the Foley catheter as a guide. Carefully insert the tracheostomy tube to be replaced. Balloon and fix the tracheostomy tube. Connect the ventilator and check the breath sounds with the stethoscope.

\section{DISCUSSION}

Data on routine tracheostomy tube change and its optimal timing are limited. Thus, the technique ensuring its safe performance is not clear. Fisher et al. (1) reported that the early group who underwent the first tracheostomy tube change within 7 days tolerated faster than the late group for speaking valve and oral intake. In addition, the short respiratory care unit and hospital stays are advantageous. Yaremchuk (2) reported that the tracheostomy tube was replaced every 2 weeks to prevent surgical intervention secondary to granulation tissue. White et al. (3) reported that performing the first tracheostomy tube change in the 1-2 weeks after tracheostomy was risky. They emphasized that it should be performed by a skilled operator in a safe environment. Tabaee et al. (4) conducted an otolaryngology training program for chief residents. During the first tracheostomy tube change, $42 \%$ of patients experienced loss of airway and $15 \%$ died.

To reduce these risks, a tracheostomy tube changer method, using a 20-cm short suction catheter and guide-wire method, a whistle tip catheter method, a thin endotracheal tube in the tracheostomy tube method, and a sterile 8-in. and 16-G catheter method were used $(3,5-7)$. The authors believe that the "Foley CatheterGuided Tracheostomy Tube Change" is a much simpler and safer method than the other methods when it is performed for the first time.

\section{Conflict of Interest Statement}

None of authors has a conflict of interest

\section{REFERENCE}

1. Fisher DF, Kondili D, Williams j, Hess DR, Bittner EA, Schmidt UH. Tracheostomy tube change before day 7 is associated with earlier use of speaking valve and earlier 
oral intake. Respir Care. 2013;58(2):257-63.

2. Yaremchuk K. Regular tracheostomy tube changes to prevent formation of granulation tissue. Laryngoscope. 2003;113(1):1-10.

3. White AC, Kher S, O'Connor HH. When to change a tracheostomy tube. Respir Care. 2010;55(8):1069-75.

4. Tabaee A, Lando T, Rickert S, Stewart MG, Kuhel WI. Practice patterns, safety, and rationale for tracheostomy tube change: a survey of otolaryngology training programs.
Laryngoscope. 2007;117(4):573-6.

5. Brwon JM, White MC. Conversion from suction catheter to guidewire for difficult tracheostomy tube change. Anaesth Intensive Care. 2004;32(6):842.

6. Mirza S, Cameron DS. The tracheostomy tube change: a review of techniques. Hosp Med. 2001;62(3):158-63.

7. Smith RB, Smith JD. A technic for tracheostomy-tube change. Anesth Analg. 1974;53(4):627-8. 